\title{
A Scoping Literature Review of Studies Assessing Effectiveness and Cost-Effectiveness of Prosthetic and Orthotic Interventions
}

\author{
Purpose: Approximately $1.5 \%$ of the world's population ( $\sim 100$ million people) \\ need a prosthesis/orthosis. The objective of the study was to establish an \\ overview of the literature that has examined prosthetic and orthotic interventions \\ with a view to inform policy development.
}

Methods: Fourteen databases were searched from 1995-2015. Studies reporting primary research on the effectiveness or cost-effectiveness of prosthetic and orthotic interventions were examined. Metadata and information on study characteristics were extracted from the included studies.

Results: The searches resulted in a total of 28,958 articles, a focus on studies with the words "randomised" OR "randomized" OR "cost" OR "economic" in their citation reduced this total to 2,644. Research has predominantly been conducted in Australia, Canada, Germany, Netherlands, UK and USA. 346 randomised controlled trials were identified, with only four randomised controlled trials examining prosthetic interventions. The majority of research examined lower limb orthoses in the adult population and used a wide range of outcome measures.

Conclusions: While various international organisations have highlighted the value of providing prosthetic and orthotic services, both to the user and society as a whole, the availability of scientific research to inform policy is limited. Future structured evaluation of prosthetic and orthotic interventions/services is warranted to inform future policy developments.

Keywords: Orthotic device, Prosthetics, Footwear, Amputation, Artificial limb, Mobility, Effectiveness, Cost-effectiveness

\section{Introduction}

About 15 out of every 100 people in the world has a disability, with between 110 and 190 million adults experiencing difficulties in activities of daily living [1]. It estimated that approximately $1.5 \%$ of the world's population (at least 100 million people) are in 
need of a prosthesis/orthosis [2]. Accurate figures on the number of people needing or accessing prosthetic and orthotic services across the world, even in developed countries, is lacking;

- In 2005, 1.6 million people in the USA were living with the loss of a limb with this figure projected to more than double by 2050 to 3.6 million [3].

- In 2006 the American Academy of Orthotists and Prosthetists projected that the number of persons using orthoses and protheses in the USA would be 7.3 million and 2.4 million, respectively, by 2020 [4].

- In 2015, the estimate for people in England treated by orthotics services was around 2 million [5].

The use of assistive technologies such as prosthetics and orthotics, when appropriate to the user and their environment, have been shown through cohort studies to increase independence and improve participation [6,7]. With the increasing older population and prevalence of many diseases, such as diabetes, there is an increasing need for prosthetics and orthotics. A report from 2002 predicted that the number of persons with an amputation, and using a prosthesis, is expected to increase by at least $47 \%$ by the year $2020(1,786,810$ people), while the number of people requiring orthoses is expected to increase by at least $31 \%(7,382,876$ people) [8].

One of the 9 recommendations made in the World Health Organization (WHO) Report on Disability is to "invest in specific programmes and services for people with disabilities" with the report stating that a review of existing programmes and services are needed [1]. The International Society for Prosthetics and Orthotics in partnership with the WHO, and funded by the US Agency for International Development (USAID), 
recently published an information product in the form of Standards for Prosthetics and Orthotic Service Provision $[9,10]$. This scoping study was conducted in order to inform these Standards, which could be used to support policy-makers to make informed decisions about the treatment and care of individuals requiring a prosthesis or orthosis.

While systematic reviews have been completed in this area, these have examined the use of prosthetic and orthotic interventions in specific clinical populations $[11,12]$, no research to date has explored and summarised the current state of research evidence across all prosthetic and orthotic interventions. This information is necessary to understand the current volume and level of evidence available for these interventions and to establish the range of clinical populations that utilise these interventions. Therefore, the aim of this study was to establish an overview of the literature that has examined prosthetic and orthotic interventions and to detail how research in this area has grown over the last 20 years. To address this aim, the focus of the study was: (1) To explore the geographical basis of the studies which evaluate the effectiveness and/or cost-effectiveness of prosthetic and orthotic interventions; (2) To identify the main languages in which these studies have been published; (3) To examine the nature of the clinical population and the medical conditions involved; (4) To investigate the types of prosthetic and orthotic devices which have been evaluated for effectiveness and/or costeffectiveness; and (5) To explore the outcome measures that have been used.

For the purposes of this article definitions for prosthesis and orthosis are as follows [13]:

Prosthesis (prosthetic device/product): externally applied device used to replace wholly, or in part, an absent or deficient limb segment (plural: prostheses). Common examples are artificial legs or hands. 
Orthosis (orthotic device/product): externally applied device used to modify the structural and functional characteristics of the neuromuscular and skeletal systems (plural: orthoses). Common examples are braces, splints and supports.

To examine the range and nature of research in this area, a scoping study design was chosen. Scoping studies differ from systematic reviews which typically focus on a well-defined question. This study design was chosen to provide breadth on the published research completed on prosthetic and orthotic provision. A scoping study addresses broader topics, it does not ask specific research questions but allows mapping of the research area and the identification of the sources and types of evidence that is available.

\section{Methods}

This study was part of a larger project which examined the scientific literature to date on prosthetic and orthotic interventions to inform the development of the Standards for Prosthetics and Orthotic Service Provision $[9,10]$.

The scoping study was performed according to the guidelines provided by Arksey and O'Malley [14]. The database searches were completed and documented following the methodology recommendations within the PRISMA guidelines for completing a systematic review [15], including the stating of the eligibility criteria, information sources, search strategy and study records.

\section{Data sources}

The following 14 databases were searched: Web of Science, Medline, PubMed, CINAHL Plus, EMBASE, SCOPUS, Rehabdata, PsycInfo, ERIC, Education Research Complete, Business Source Complete, IEEE, NIHR and CEA Registry. MeSH headings 
and free text terms for orthotics and prosthetics were used along with study design categories to capture all research in the area of orthotics and prosthetics (sample search strategy for MEDLINE is provided elsewhere [16].. No language restriction was applied to the search. Searches were adapted for each database and were completed between 22nd and 27th September 2015.

\section{Study selection}

Initially, for the purpose of the wider project, results were screened to identify all studies examining prosthetic and orthotic interventions. Subsequently, these results were further screened the locate the studies included in this scoping review. A flow diagram for study selection is provided in Figure 1.

Two reviewers independently screened the search results. Inclusion criteria were as follows, with studies only included if they met all criteria:

- Studies which provided devices (orthosis/prosthesis) for a clinical problem and for use during activities of daily living

- Studies which involved participants off any age and any medical condition

- Studies which used valid outcome measures

Exclusion criteria were as follows:

- Case series/report/studies; expert opinion articles; letters to the editor; commentaries; cross-sectional studies

- Studies involving healthy participants 
- Studies examining devices for prevention of injuries and within therapy/training sessions

- Studies examining novel/research devices.

The independent screening was completed using the online screening software provided by Covidence (https://www.covidence.org/), with discrepancies between reviewers resolved through discussion.

Following the initial title only screening of the search results a high volume of articles $(9,228)$ remained for title and abstract screening. Due to the large volume of studies it was decided to focus on locating the studies with the highest level of evidence (Randomised Controlled Trials (RCTs); OCEBM Levels of Evidence [17]) and those which examined cost-effectiveness. To locate these studies an "All field" search within Endnote X7 software (Thomas Reuters, USA) of the 9,228 results for the words "randomised" OR "randomized" OR "cost" OR “economic" was conducted. This search yielded 2,639 results, which with the additional 5 records identified through other sources, resulted in a total of 2,644 articles for title and abstract screening.

\section{Data extraction}

Following screening, data extraction was completed on the articles which met the inclusion criteria, with additional detailed data extraction completed on the identified RCTs. Three reviewers extracted data regarding the characteristics of the included studies, with the extracted data checked for accuracy and completeness by a second reviewer. Any identified discrepancies were then discussed by the reviewers to ensure the accuracy of extracted data.

For articles which met the inclusion criteria metadata including the 
corresponding author's affiliation (country), year of publication, and publication language were extracted. Additionally, for the subset of RCTs the extracted characteristics from each study also included the clinical population examined, the type of orthotic/prosthetic intervention, the comparator/s and the outcome measures used.

\section{Data synthesis}

Extracted data was synthesised descriptively with studies grouped based on their study design and the clinical population of the participants.

\section{Results}

The database searches resulted in a total of 28,958 articles (Figure 1) and the results were stored and managed in Endnote X7 software (Thomas Reuters, USA). Initial screening by two reviewers reduced the total to 9,228. The search for RCTs and studies examining cost-effectiveness reduced the total to 2,639 . When these results were screened, published protocols were identified from which an additional 5 potential studies were added for screening, giving a total of 2,644 studies.

\section{Figure 1 near here}

Metadata analysis of the 919 included studies was completed to gain an understanding of how research in this area has progressed over the last 20 years and to identify the countries where this research was completed and the languages in which studies were published. There has been a steady rise in the number of publications per year examining orthoses (total of 878 studies) with a limited number of studies examining prostheses (total of 41 studies) (Figure 2). Analysis of the RCT subset of results also showed that there has been an increase in RCTs examining orthoses 
completed in this area over the last 20 years, however only four RCTs examining prostheses were identified (Figure 3).

Figure 2 near here

Figure 3 near here

(1) Exploration of the geographical basis of the studies which evaluate the effectiveness and/or cost-effectiveness of prosthetic and orthotic services/interventions

Analysis of the 878 studies which examined orthoses identified that the UK and USA were the countries which published the highest volume of articles in this area with over 100 articles from each country (Table 1). For this analysis, the countries were identified as the country affiliation provided by the corresponding author of the article. Of the 41 studies which examined prostheses 12 were affiliated to the USA, with 6 each from the UK and The Netherlands, 4 studies from Italy, 2 studies each from Canada, France, Japan and Sweden and 1 study each from India, Iran, Malaysia, Nigeria and Turkey.

Table 1 near here

(2) Identification of the main languages of the studies which evaluate the effectiveness and/or cost-effectiveness of prosthetic and orthotic services/interventions.

A breakdown of the languages in which these 919 studies were published are provided in Figure 4. Majority of the articles were published in English (92\% of results) followed by German (3\%), and the remaining $5 \%$ of the results consisted of a small number of articles in 13 other languages (Turkish, French, Spanish, Dutch, Portuguese, Chinese, Czech, Farsi, Hebrew, Croatian, Danish, Italian and Swedish, listed in order of frequency from highest to lowest). For the 41 prosthetic intervention studies, 38 were 
published in English with 1 study each in Farsi, Italian and Turkish.

Figure 4 near here

Of the 919 studies included in this review, 346 were RCTs (RCT subset), of which 323 were published in the English language. References for the 346 RCTs are provided in Supplemental Files A-C. The clear majority of the RCTs examined orthotic interventions with only 4 studies examining prosthetic interventions.

In addition to the 323 RCTs which published in the English language there were 23 non-English language RCTs (German (11 studies), Turkish (5 studies), Dutch (2 studies), 1 study each in Chinese, Czech, Danish, French and Spanish) identified through the search (see Supplemental Files B for references). None of these non-English language RCTs examined prosthetics provision.

(3) Examination of the nature of the clinical population and the medical conditions involved in the studies which evaluate the effectiveness and/or cost-effectiveness of prosthetic and orthotic services/interventions.

The examination of clinical populations and medical conditions was completed on the RCT subset of results. Of the 346 RCTs only 4 examined prosthetic interventions and all studies investigated lower limb prostheses. These studies compared lower limb socket systems, and none investigated cost-effectiveness.

The majority of the 342 RCTs investigating orthotics interventions studied the adult population (87\% of studies) and examined lower limb orthoses (57\% lower limb; 30\% upper limb; $12 \%$ spine; and $1 \%$ head). There was a large range of clinical populations/medical conditions examined in these included studies. Most adult 
population studies examined the provision of orthotic interventions for people with osteoarthritis (wrist/hand splint; knee brace; foot orthosis; footwear), fractures (brace/splint) and stroke (Ankle foot orthosis (AFO); wrist/hand splint/brace; function electrical stimulation (FES)). The majority of the studies which examined the paediatric population focused on cerebral palsy (AFO; lycra garment; foot orthosis; SWASH brace). There were 34 studies which examined a paediatric population, with other medical conditions examined including fractures, scoliosis and arthritis. Only 5 of the 342 RCTs examining orthotics provision examined the cost-effectiveness of the tested interventions.

(4) Investigation of the types of prosthetic and orthotic devices which have been evaluated for effectiveness and/or cost-effectiveness.

The examination of devices was completed on the RCT subset of result. The limited number of only four RCTs investigating prosthetics all examined lower limb socket systems and none investigated cost-effectiveness. The RCTs investigating orthotic interventions examined footwear, foot orthoses, wedges, ankle foot orthoses (AFOs), knee ankle foot orthoses (KAFO); thoraco lumbo sacral orthoses (TLSO), supports/belts, splints, braces, orthotic garments (e.g. lycra), helmets and cervical collars. The five RCTs which investigated cost-effectiveness examined braces, splints, supports and foot orthoses.

(5) Exploration of the outcome measures that have been used in studies which evaluate the effectiveness and/or cost-effectiveness of prosthetic and orthotic 
services/interventions.

The examination of outcome measures was completed on the RCT subset of result. The included RCTs used a wide range of outcome measures to examine effectiveness of interventions. Questionnaires and scales were utilised to assess pain, quality of life, and functional improvements (e.g. SF-36, Cincinnati knee score, Lysholm Knee Score, WOMAC pain score, Oswestry Disability Index, Foot Health Status Questionnaire and Gross Motor Function Measure (GMFM) score, Manchester Foot Pain and Disability Questionnaire and DASH (Disabilities of arm, shoulder and hand)). Clinical quantitative measures such as walking speed, functional reach and timed up and go tests and Cobb angle were used. Some studies utilised biomechanical (e.g. range of motion, torque, grip strength, plantar pressure) and physiological (Physiological Cost Index) quantitative assessments. To assess cost-effectiveness, the RCTs utilised incremental cost-effectiveness ratios, bootstrapping analysis and cost-effectiveness acceptability curves.

\section{Discussion}

The results of this scoping study show that research in this area has grown substantially in the last 20 years, with the majority of this research conducted in a small number of countries. The vast amount of this research focused on lower limb orthotics in the adult population, with scarce RCTs examining lower limb prostheses and none investigating upper limb prostheses. No RCTs were identified which examined service provision, with all studies examining the provision of specific prosthetic and orthotic interventions. As these RCTs examine an extensive range of specific interventions the capacity to synthesis their findings to inform policy is challenging. 
The identified RCTs used a large variety of outcome measures examining different aspects of function, activity and participation. This complicates synthesis of data from studies which examined the same prosthetic or orthotic service/intervention but used different outcomes measures or different scales to measure the same outcome measure. While the scientific literature has yet to provide high quality research into prosthetics and orthotics service provision various organisations (for example the British Health Trade Association and the National Health Service in the UK $[5,6,18,19]$ and the American Orthotic and Prosthetic Association and The Amputee Coalition in the USA [20] have commissioned reports into these services. These reports, while not presented within our results as they are not included in scientific databases, highlight the positive impact these services have on users (improvements in quality of life and greater independence) and the potential cost saving in healthcare that can be achieved when these services are implemented correctly. Furthermore, the importance of orthoses and prostheses has been highlighted by the inclusion of club foot braces, lower/upper limb and spinal orthoses, lower limb prostheses and therapeutic footwear in the recently produced WHO Priority Assistive Products List [21]. This list is intended to promote access to assistive technology to enable those who require them to live healthy, productive and dignified lives. Provision of such a list and its adoption by various Government and NonGovernmental agencies has the potential for structured research and development into, not only the development of these products but also the service provision. At this point, one should highlight that this research has to be conducted at the point of delivery with socio cultural and related biomechanical requirements taken into consideration. This will help to develop focussed and cost-effective service provision.

The main emphasis of research to date in this area has been to examine the effectiveness of specific prosthetic or orthotic interventions, however, it is vital that future 
research also examines cost-effectiveness. Information on cost-effective prosthetic/orthotic interventions is needed, as this will provide an evidence base for their provision to healthcare providers and policy makers. While our search found no RCTs which examined cost-effectiveness of prosthetic provision a study recently published in a Special Issue of Military Medicine [22] analysed Medicare claims data and found that individual who received lower extremity prostheses had comparable Medicare episode payments and better outcomes than those who did not receive prostheses. Individuals who were provided with prostheses were more likely to receive extensive outpatient therapy; and receiving physical therapy was associated with fewer hospitalisations and emergency room visits, and less facility-based care. The authors concluded that these reductions in hospital costs in those who received prostheses essentially offset the cost of the prosthetic over a 12-month time frame. Future prospective studies are needed to examine the costeffectiveness of prosthetics and orthotics service provision.

Although one could argue that the musculoskeletal chronic health needs of the world is similar in all types of economies, one would appreciate that the needs of the developing and the underdeveloped regions of the world would be different to that of a developed country. Whilst in all cases people would be looking for solutions to help alleviate chronic pain resulting from musculoskeletal disorders, one group will look at achieving a better life style and the other will look for less expensive solutions with a view to meet the basic socio-economic needs. This no doubt has implications for global health policies.

Around the world, the prevalence of musculoskeletal disorders is high and they are identified as the $2^{\text {nd }}$ highest cause of the morbidity-related global burden of disease (Global Burden of Disease (GBD) study: http://www.healthdata.org/gbd). This high prevalence could be attributed to the fact that musculoskeletal system disorders 
encompass a vast range of disorders, ranging from conditions which have developed due to lifestyle factors at one end to those amputations as a result of military conflicts at the other end. However, in general, there has been an inadequate response in terms of policy and funding $[23,24]$ to prevent and treat these disorders. The focus has been on other significant global health issues such as cancer HIV/AIDS and cardiovascular diseases. At present, there is not a coordinated and coherent research and development strategy in this area, with different organisations and research groups examining specific treatment interventions and not examining the global or local needs.

Whilst, this study provides a general overview on the breadth of high-level published research which has examined the effectiveness and cost-effectiveness of prosthetic and orthotic provision, further in-depth analysis of the studies identified within this scoping study needs to be completed. Following on from the findings of this scoping study, a systematic review on prosthetic and orthotic interventions has been published [16].

\section{Conclusions}

While various international organisations have highlighted the value of providing Prosthetic and Orthotic services both to the user and society as a whole the availability of scientific research to inform policy development is limited. The majority of this limited research examined lower limb orthoses in the adult population and used a wide range of outcome measures. There is a clear need to address global health systems and to examine health systems as a whole entity as opposed to a disease/condition specific service. 


\section{Limitations}

The literature search in this study was based on scientific databases, we did not include grey literature in which may have limited the results. The grey literature may provide relevant information not evident in the scientific literature.

While the database searches for the wider project resulted in a large volume of articles, this study focused on studies with highest level of evidence (RCTs) and those examining cost-effectiveness of prosthetic and orthotic interventions. Utilising a randomised controlled trial design for these interventions is challenging which resulted in a relatively low number of studies included in this scoping study compared to the original number of total results.

Acknowledgements

The authors gratefully acknowledge the support of the advisory panel who supported this work: Anil Kumar Jain, Santokba Durlabhji Hospital, Jaipur, India; Elizabeth Hardin, Louis Stokes Cleveland DVA Medical Center, USA; Géza Kogler, Georgia Institute of Technology, Atlanta, USA; Isabel Sacco, University of Sao Paulo, Brazil; Saeed Zahedi, Chas A Blatchford \& Sons, UK; Sarah Curran, Cardiff Metropolitan University, UK; Stefano Negrini, Italian Scientific Institute for the Spine, Italy; Zulfiqarali G. Abbas, Abbas Medical Centre, Tanzania. We also acknowledge our colleagues Dave Parkes and Paul Biggs, Staffordshire University, UK and Mathew Stone, Royal Stoke University Hospital, UK who supported the development of the database search strategies.

References:

[1] World Health Organization.World Report on Disability Geneva, Switzerland. ; 2011; Available from: http://www.who.int/disabilities/world_report/2011/en/.

[2] Khasnabis C.Standards for Prosthetics and Orthotics Service Provision: 2015-2017 work plan. Version 4 2015; Available from: http://www.who.int/phi/implementation/assistive_technology/workplan_po_standards.pdf.

[3] Ziegler-Graham K, MacKenzie EJ, Ephraim PL, Travison TG, Brookmeyer R. Estimating the Prevalence of Limb Loss in the United States: 2005 to 2050. Archives of Physical Medicine and Rehabilitation 2008;89:422-9.

[4] Prosthetists AAoOa. O\&P trends and statistics. 2006. 
[5] NHS England.Improving the Quality of Orthotics Services in England; 2015; Available from: https://www.england.nhs.uk/commissioning/wpcontent/uploads/sites/12/2015/11/orthcs-final-rep.pdf.

[6] Hutton JL, Hurry M.Orthotic Service in the NHS - Improving Service Provision; 2009.

[7] Samuelsson KA, Toytari O, Salminen AL, Brandt A. Effects of lower limb prosthesis on activity, participation, and quality of life: a systematic review. Prosthet Orthot Int 2012;36:145-58.

[8] Nielsen C.Issues Affecting the Future Demand for Orthotists and Prosthetists: Update 2002 National Commission on Orthotic and Prosthetic Education; 2002; Available from: http://www.ncope.org/summit/pdf/Footnote3.pdf.

[9] World Health Organization.WHO standards for Prosthetics and Orthotics. Part 1. Standards; 2017; Available from: http://www.who.int/phi/implementation/assistive_technology/prosthetics_orthoti cs/en/.

[10] World Health Organization.WHO standards for Prosthetics and Orthotics. Part 2. Implementation manual; 2017; Available from: http://www.who.int/phi/implementation/assistive_technology/prosthetics_orthoti cs/en/.

[11] Cumming J, Barr S, Howe Tracey E. Prosthetic rehabilitation for older dysvascular people following a unilateral transfemoral amputation. Cochrane Database of Systematic Reviews 2015.

[12] Lewis J, Lipp A. Pressure-relieving interventions for treating diabetic foot ulcers. Cochrane Database Syst Rev 2013;1:CD002302.

[13] International Organization for Standardization. Prosthetics and orthotics Vocabulary - Part 1: General terms for external limb prostheses and external orthoses. Geneva, Switzerland. 1989.

[14] Arksey H, O'Malley L. Scoping studies: towards a methodological framework. International Journal of Social Research Methodology 2005;8:19-32.

[15] Moher D, Liberati A, Tetzlaff J, Altman DG. Preferred reporting items for systematic reviews and meta-analyses: The PRISMA statement. PLoS Medicine 2009;6:e1000097.

[16] Healy A, Farmer S, Pandyan A, Chockalingam N. A systematic review of randomised controlled trials assessing effectiveness of prosthetic and orthotic interventions. PLOS ONE 2018;13:e0192094.

[17] OCEBM Levels of Evidence Working Group.The Oxford 2011 Levels of Evidence; 2011; Available from: http://www.cebm.net/index.aspx?o=5653.

[18] Business Solutions.Orthotic Pathfinder Report; 2004; Available from: http://www.bhta.net/sites/default/files/documentupload/2012/orthotic_pathfinder_report_july_2004.pdf.

[19] Centre for Economics and Business Research.The economic impact of improved orthotic services provision; 2011; Available from:

http://www.bhta.net/sites/default/files/documentupload/2012/Orthotics_review_Cebr_report_04_07_2011.pdf.

[20] Dobson DaVanzo \& Associates.Retrospective Cohort Study of the Economic Value of Orthotic and Prosthetic Services Among Medicare Beneficiaries; 2013; Available from: http://www.aopanet.org/resources/research/.

[21] World Health Organization.Priority Assistive Products List; 2016; Available from: http://www.who.int/phi/implementation/assistive_technology/EMP_PHI_2016.0 $\underline{1 / \mathrm{en} / .}$ 
[22] Dobson A, El-Gamil A, Shimer M, DaVanzo JE. Economic Value of Prosthetic Services Among Medicare Beneficiaries: A Claims-Based Retrospective Cohort Study. Mil Med 2016;181:18-24.

[23] Mock C, Cherian MN. The Global Burden of Musculoskeletal Injuries: Challenges and Solutions. Clinical Orthopaedics and Related Research 2008;466:2306-16.

[24] Woolf AD. Global burden of osteoarthritis and musculoskeletal diseases. BMC Musculoskeletal Disorders 2015;16:S3-S. 
Table 1. List of countries by number of articles examining orthotic interventions.

\begin{tabular}{|c|c|c|c|}
\hline Number of articles & Country (corre & onding author & ation) \\
\hline 100-194 & $\begin{array}{l}\text { UK } \\
\text { USA }\end{array}$ & & \\
\hline $50-99$ & $\begin{array}{l}\text { Australia } \\
\text { Canada }\end{array}$ & $\begin{array}{l}\text { Germany } \\
\text { The Netherlar }\end{array}$ & \\
\hline $10-49$ & $\begin{array}{l}\text { Brazil } \\
\text { China } \\
\text { Denmark } \\
\text { France }\end{array}$ & $\begin{array}{l}\text { India } \\
\text { Italy } \\
\text { New Zealand } \\
\text { Norway }\end{array}$ & $\begin{array}{l}\text { Sweden } \\
\text { Switzerland } \\
\text { Turkey }\end{array}$ \\
\hline$<9$ & $\begin{array}{l}\text { Austria } \\
\text { Bangladesh } \\
\text { Belgium } \\
\text { Chile } \\
\text { Colombia } \\
\text { Croatia } \\
\text { Czech Republic } \\
\text { Egypt } \\
\text { Ethiopia } \\
\text { Finland }\end{array}$ & $\begin{array}{l}\text { Greece } \\
\text { Hong Kong } \\
\text { Iran } \\
\text { Ireland } \\
\text { Israel } \\
\text { Japan } \\
\text { Lebanon } \\
\text { Mexico } \\
\text { Pakistan } \\
\text { Qatar }\end{array}$ & $\begin{array}{l}\text { Saudi Arabia } \\
\text { Singapore } \\
\text { Slovenia } \\
\text { South Africa } \\
\text { South Korea } \\
\text { Spain } \\
\text { Taiwan } \\
\text { Thailand }\end{array}$ \\
\hline
\end{tabular}




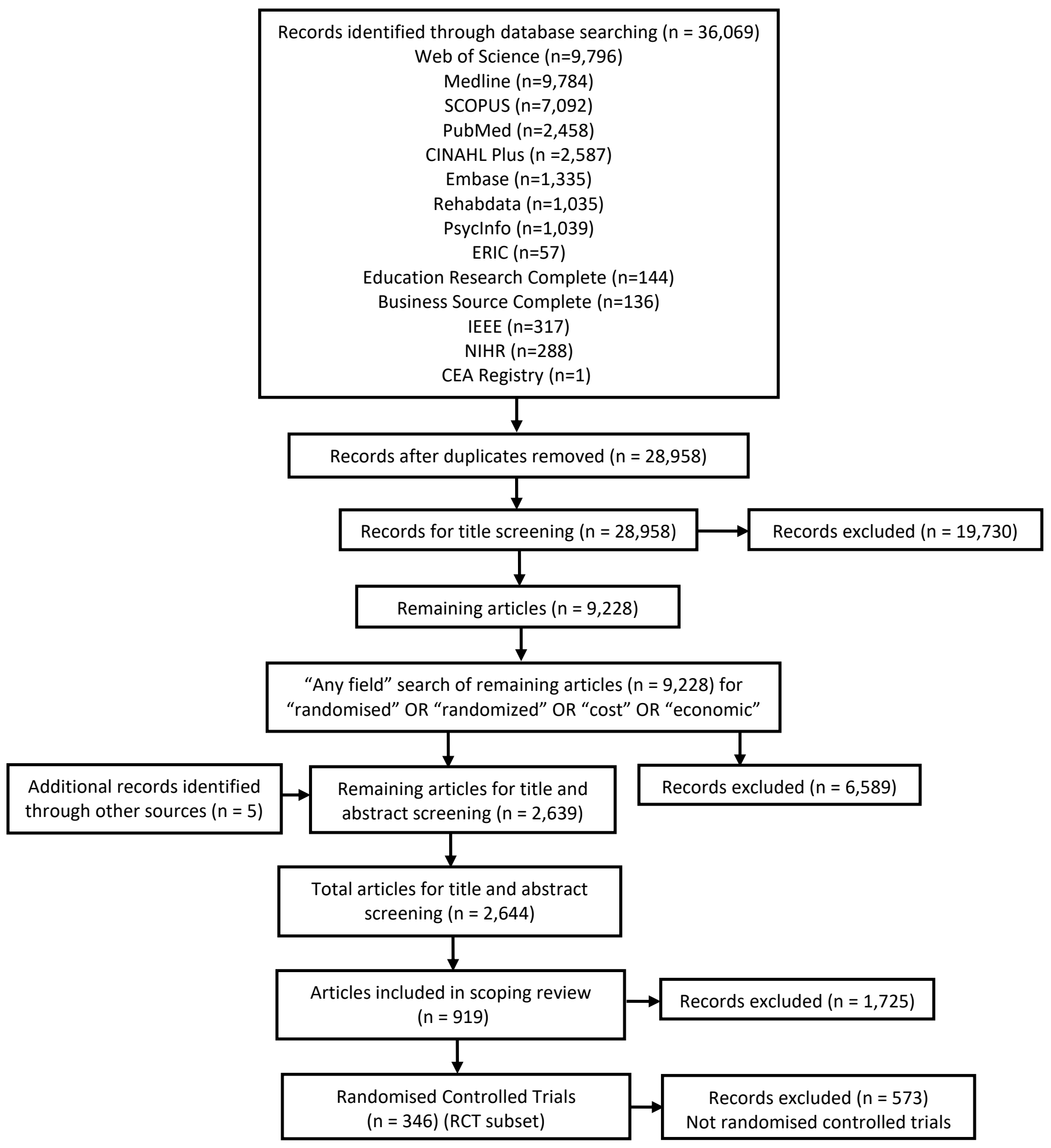

Figure 1. Flow diagram for study selection. 


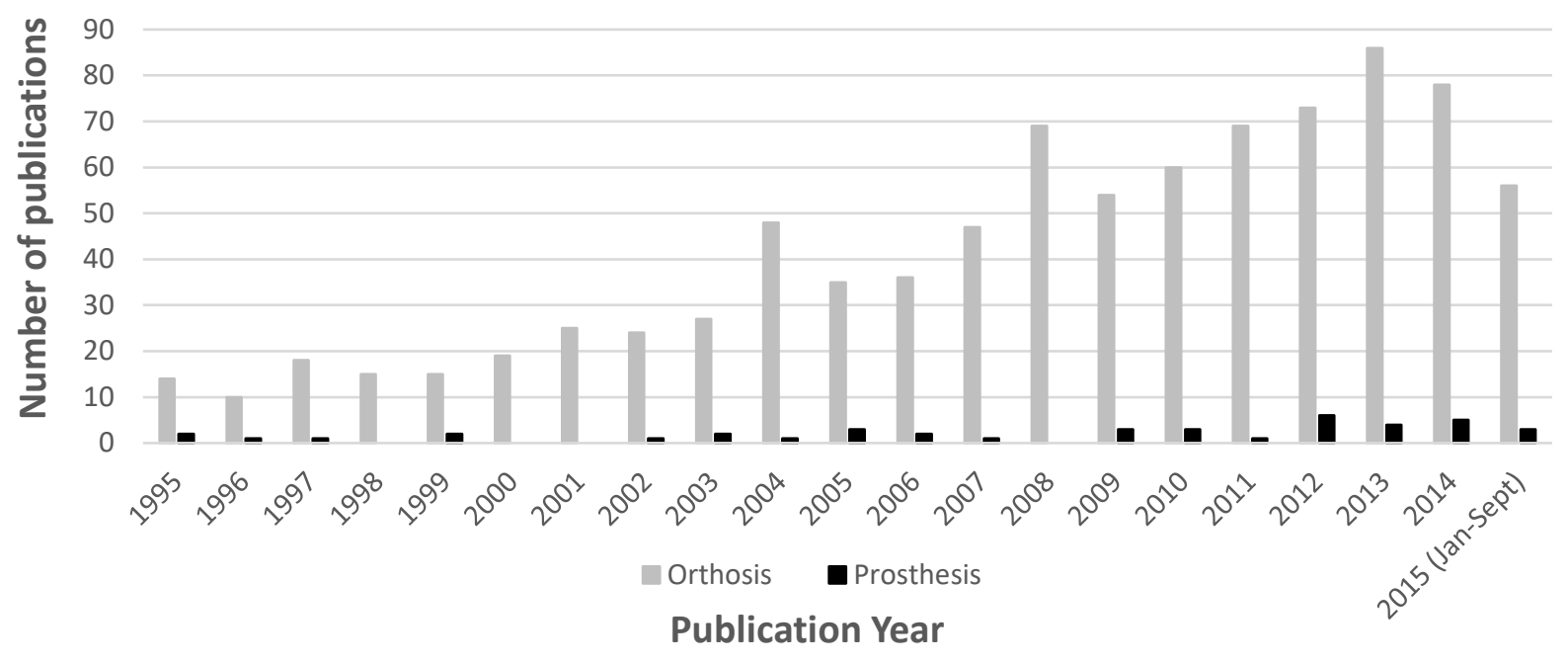

Figure 2. Number of publications per year for 20 years (January 1995 - September 2015). 


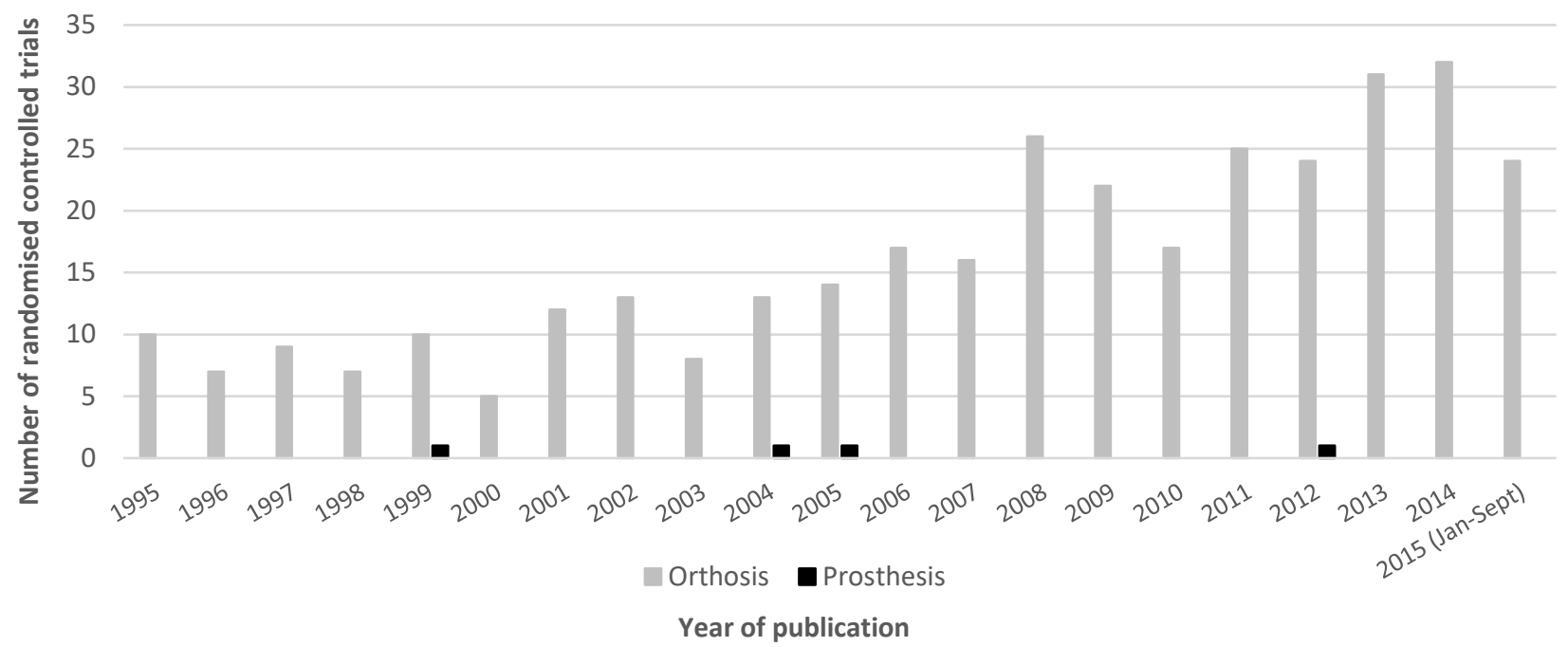

Figure 3. Number of randomised controlled trials per year for 20 years (January 1995 September 2015). 


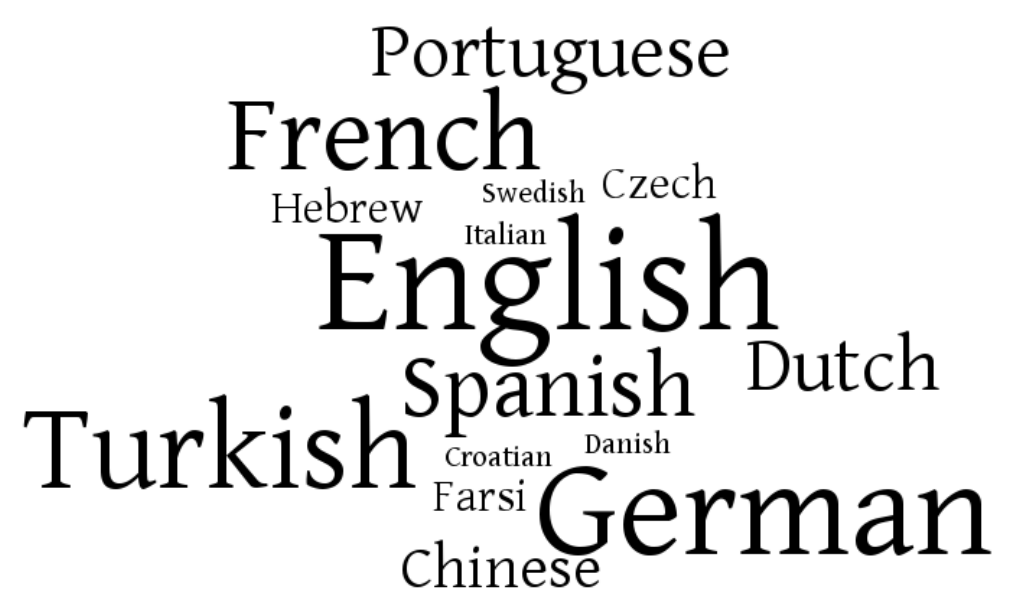

Figure 4. Word cloud representing publication languages (size of each term is proportional to its representation in the review). 